\section{Melanom-Indizien am Nagel}

\section{Vieles kann eine Melanonychie verursachen. Bei bestimmten Hinweisen ist} das Risiko für ein Melanom allerdings erhöht.

$\mathrm{T}$ raumata, Medikamente wie Zidovudin oder Zytostatika, Endokrinopathien, Onychomykosen oder Veränderungen im Rahmen einer Schwangerschaft können zu Verfärbungen der Nägel führen. Doch bei einigen Hinweiskonstellationen sollte man wegen der Melanomgefahr aufmerksam werden, erläuterte Prof. Carola Berking, Hautklinik der LMU, München. Liegen anamnestisch keine Hinweise auf ein Trauma oder ver-

\section{ABCDEF-Regel \\ A Alter / Afroamerikaner \\ B Band / Breite $>3 \mathrm{~mm}$ \\ C Change (Veränderungen) \\ D Digit - Lokalisation häufig am \\ D1 von Hand bzw. Fuß \\ E Extension (Hutchinson) \\ $\mathrm{F}$ Familienanamnese / Eigen- anamnese}

Nach Levit EK et al. J Am Acad Dermatol 2000; 42: 269-74 dächtige Medikamente vor und besteht die Verfärbung schon länger und verändert sich über die Zeit, ist insbesondere bei einem Melanom in der Vorgeschichte an ein erneutes Melanom zu denken. Besonders häufig betroffen ist der Daumen (18-31\%) - meist der dominanten Hand - oder der Großzeh (24-43\%). Weitere Indizien sind eine Beteiligung der Matrix und ein eher höheres Alter der Patienten. Die Nagelplatte weist häufig Fissuren auf, ist verdünnt, es treten Ulzeration auf und der Nagel ist oft destruiert.

\section{Breit ist schlecht}

Aufmerksam machen sollten insbesondere über 3 bis $5 \mathrm{~mm}$ breite Pigmentierungen, solche die nach distal schmaler werden, inhomogene und unscharf begrenzte Streifen sowie das Auftreten von Hutchinson-Zeichen, wobei auch der distale Hautbereich unter dem Nagel betroffen sein kann. Eine Melanonychia striata ist laut Berking in 76\% der Fälle vorhanden. In der Dermatoskopie mit Ultra-

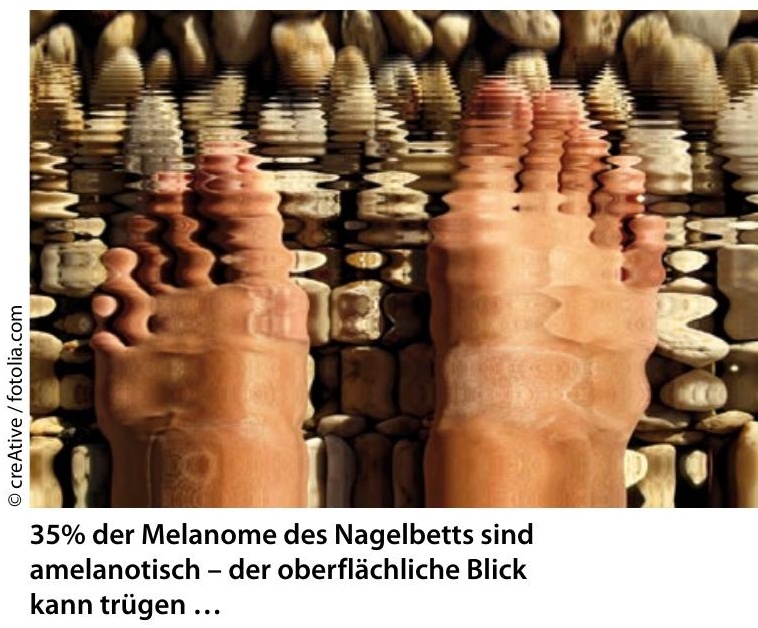

schallgel zeigen sich braune und braunschwarze Strukturen, eine inhomogene Verfärbung mit unregelmäßigen Linien und Punkten und atypische Gefäße. Kurzgefasst gilt die ABCDEF-Regel (vergl. Kasten). Aber eins sollte man bei all dem nicht vergessen, betonte Berking: $35 \%$ der Melanome des Nagelbetts sind amelanotisch.

Friederike Klein

Berking C. Pigmentierungen und Neoplasien der Nägel - wann biopsieren oder exzidieren?

\title{
Lokalanästhesie und mehr: Nützliches für die Nagelchirurgie
}

\section{Dr. Dorothee Dill-Müller, Hautklinik am Klinikum Lüdenscheid, gab in ihrem Vortrag Einblicke darin, was bei einem chirurgischen Eingriff am Nagel beachtet werden muss.}

ei Eingriffen am Nagel kommt meist eine Leitungsanästhesie nach Oberst zum Einsatz. In der Regel reichen 3-4 ml Lokalanästhetikum, am Großzeh kann es auch 5-6 ml erfordern. Verwendet werden Mecain $1 \%$ oder Lidocain 2\%. „Sie können auch, um Ihre Patienten postoperativ schmerzfrei zu halten, lang wirkende Lokalanästhetika verwenden“, sagte Dr. Dorothee DillMüller aus Lüdenscheid. In solch einem Fall werden 2-3 ml Bupivacain oder Ropivacain $0,2 \%$ indiziert.

Auch ein Adrenalin-Zusatz ist möglich. „Es gibt keine wissenschaftliche Evidenz, dass dadurch das Nekroserisiko an den Akren erhöht wäre“, erklärte Dill-Müller. Dies konnte in den letzten Jahren in mehreren Publikationen belegt werden. DillMüller warnte jedoch davor, eigene Mischungen herzustellen. Vielmehr sollten Fertigpräparate mit der Verdünnung 1:100.000-200.000 verwendet werden. Bei Risikopatienten mit ungeklärter Thrombophilie, Thromboseneigung, Vasospasmen oder Patienten, die entsprechende Medikamente einnehmen, sollte auf die Zugabe von Adrenalin verzichtet werden.

Eine Blutsperre sollte nach 15-20 Minuten gelöst werden. Die meisten Ein- griffe sind nach dieser Zeit aber sowieso abgeschlossen. Eine Elektrokoagulation wird laut Dill-Müller sehr selten benötigt. „Versuchen Sie sie zu vermeiden.“ Eine Blutung könne postoperativ vermieden werden, indem der Patient die Extremität hoch lagert.

Das weitere postoperative Verhalten des Patienten ist im Prinzip genauso wichtig wie die richtigen ärztlichen Techniken während des Eingriffs. Neben der Hochlagerung gehören dazu eine Nikotinkarenz von vier bis fünf Tagen sowie eine Analgesie für ein bis zwei Stunden postoperativ. „In der Regel benötigen Sie keine antibiotische Prophylaxe - außer bei Patienten mit Diabetes oder hochgradiger peripherer arterieller Verschlusskrankheit", sagte Dill-Müller abschließend.

Dr. Nicola Zink

Dill-Müller D. Nagelchirurgie. 